\title{
CUSTOS DE SUPRIMENTOS: ESTUDO EXPLORATÓRIO COM APLICAÇÃO DE MODELO DE MENSURAÇÃO DE ÇUSTOS LOGÍSTICOS
}

\author{
SUPPLIES COSTS: AN EXPLORATORY STUDY \\ WITH APPLICATION OF MEASUREMENT \\ MODEL OF LOGISTICS COSTS
}

Recebido 20-09-2012

Aceito 10-12-2012

Ana Paula Ferreira Alves ${ }^{1}$ José Vanderlei Silva Borba ${ }^{2}$ Gilberto Tavares dos Santos ${ }^{3}$ Artur Roberto Gibbon ${ }^{3}$

\section{RESUMO}

Uma das principais razões da dificuldade na adoção de um método integrado de apuração de custos logísticos é a falta de informação apropriada sobre esses custos. O gerenciamento da cadeia logística e a identificação de seus custos podem proporcionar informações para seus gestores no que diz respeito à tomada de decisão, gerando vantagem competitiva. Alguns modelos de apuração de custos logísticos são propostos por Uelze (1974), Dias (1996), Goldratt (2002), Christopher (2007), Castiglioni (2009) e Borba e Gibbon (2009), com escassa divulgação dos resultados obtidos. Nesse contexto, este estudo objetiva avaliar os custos de suprimentos, por meio de um modelo de mensuração de custos logísticos. Metodologicamente, o estudo caracteriza-se como exploratório. O modelo aplicado apontou, na condição original, que estavam sendo aplicados cerca de 2,5 milhões de reais no processo de gestão dos suprimentos, com custos de reposição e de armazenagem em desequilíbrio. Readequando os dados da empresa, é possível obter $52 \%$ de redução dos custos de repor e de armazenar suprimentos. Assim, o modelo aplicado de custos logísticos aos suprimentos mostrou viabilidade de implantação, bem como revelou dispor de elementos para auxiliar na forma de gestão e de tomada de decisão na área de logística de suprimentos.

Palavras-chave: Custos; logística; modelo de mensuração; suprimentos.

\footnotetext{
${ }^{1}$ Possui graduação em Administração e em Ciências Contábeis pela Universidade Federal do Rio Grande - FURG. Atualmente é mestranda em Administração na Universidade Federal do Rio Grande do Sul - UFRGS. Porto Alegre, Rio Grande do Sul, Brasil. E-mail: anapfalves@gmail.com.

${ }^{2}$ Possui graduação em Administração e especialização em Contabilidade pela Universidade Federal do Rio Grande do Sul - FURG, especialização em Metodologia do Ensino Superior pela Universidade Federal do Rio Grande do Sul - UFRGS e doutorado em Integração Regional pela Universidade Federal de Pelotas - UFPEL. Atualmente é professor adjunto da Universidade Federal de Rio Grande - FURG. Rio Grande, Rio Grande do Sul, Brasil. E-mail: vanderleiborba@vetorial.net.

${ }^{3}$ Possui graduação em Administração e mestrado e doutorado em Engenharia de Produção pela Universidade Federal do Rio Grande do Sul - UFRGS. Atualmente é professor adjunto na Universidade Federal do Rio Grande do Sul - UFRGS. Porto Alegre, Rio Grande do Sul, Brasil. E-mail: gilbertotav@hotmail.com.

${ }^{4}$ Possui graduação em Ciências Contábeis pela Universidade Federal do Rio Grande - FURG e mestrado em Administração pela Universidade Federal de Santa Catarina - UFSC. Atualmente é professor assistente da Universidade Federal do Rio Grande - FURG. Rio Grande, Rio Grande do Sul, Brasil. E-mail: argibbon@uol.com.br.
} 


\begin{abstract}
One of the main reasons for the difficulty in adopting an integrated method of calculation of logistics costs is still a lack of adequate information about costs. The management of the supply chain and identify its costs can provide information for their managers, with regard to decision making, generating competitive advantage. Some models of calculating logistics costs are proposed by Uelze (1974), Dias (1996), Goldratt (2002), Christopher (2007), Castiglioni (2009) and Borba \& Gibbon (2009), with little disclosure of the results. In this context, this study aims to evaluate the costs of supplies, applying a measurement model of logistics costs. Methodologically, the study characterized as exploratory. The model applied pointed, in original condition, that about $R \$ 2.5$ million were being applied in the process of management of supplies, with replacement costs and storage imbalance. Upgrading the company's data, it is possible obtain a $52 \%$ reduction in costs to replace and store supplies. Thus, the cost model applied to logistical supplies showed feasibility of implementation, as well as providing information to assist in management and decision-making in logistics supply.
\end{abstract}

Keys words: Costs; logistics; measurement model; supply.

\title{
1 INTRODUÇÃO
}

Uma das principais razões de existir dificuldade na adoção de um método integrado para apuração de custos logísticos em muitas empresas é a falta de informação apropriada sobre tais custos. Os componentes da cadeia logística (suprimentos, produção, distribuição) apontam para a necessidade de apuração gerencial de custos, visto que os custos logísticos são responsáveis por grande parcela dos custos totais de uma empresa (BALLOU, 2001). É notória a importância do gerenciamento dos custos logísticos, entretanto, o que se percebe na prática gerencial indica que os tomadores de decisões das empresas determinam custos de maneira empírica, reunindo os custos de suprimentos e de distribuição aos custos de produção, o que acaba por interferir diretamente no resultado financeiro.

Os sistemas contábeis convencionais geralmente não contribuem para a identificação dos impactos dos custos relativos à logística, uma vez que, com frequência, alocam-nos em outras áreas de custo. $O$ gerenciamento da cadeia logística e a identificação de seus custos podem proporcionar informações específicas para seus gestores, no que diz respeito à tomada de decisão (CHRISTOPHER, 2007). Assim, é possível constatar que a gestão dos custos é um dos fatores capazes de proporcionar competitividade de forma direta à organização, através da produção bens e/ou da prestação serviços a custos mais baixos do que os concorrentes conseguem administrar (PIANA; ERDMANN, 2011). De acordo com Christopher (2007), a fonte da vantagem competitiva de uma empresa está, primeiramente, na capacidade da organização de diferenciar-se de outras e, secundariamente, de operar com custos menores. Nessa perspectiva, pode-se afirmar que não basta apenas controlar os custos, estes devem ser quantificados para que possam ser devidamente gerenciados (LOPES et al, 2013). Modelos de apuração de custos logísticos são contemplados em propostas de Uelze (1974), Dias (1996), Goldratt (2002), Christopher (2007), Castiglioni (2009) e Borba e Gibbon (2009). Entretanto, existem modelos com maior ou menor aderência à mensuração de custos logísticos, uma vez que as variáveis dos diferentes modelos oscilam em nomenclatura e abrangência. Além disso, os modelos apresentam limitada divulgação dos resultados obtidos. Desse modo, surge a questão de pesquisa: a aplicação de um modelo de apuração de custos logísticos, em uma organização, pode fornecer elementos de gestão para os tomadores de decisão?

A seleção do modelo baseou-se na cobertura de custos de suprimentos e de custos de distribuição. No entanto, a identificação de um modelo aplicável à realidade de pesquisa exigiu 
o detalhamento de quais custos da cadeia logística deveriam ser abordados. Diante dessa realidade, optou-se por desdobrar o modelo de custos logísticos, utilizando, na análise, os cálculos referentes aos custos de suprimentos. Nesse contexto, este estudo possui como objetivo avaliar os custos de suprimentos por meio da aplicação de um modelo de mensuração de custos logísticos. Para tanto, foi necessário viabilizar as informações de custos de uma empresa para testar o modelo selecionado.

Este trabalho está dividido em quatro partes, além desta introdução. Na segunda, foi realizada uma revisão da literatura acerca dos custos empresariais, da cadeia e dos custos logísticos e dos modelos de mensuração de custos. Na terceira, está descrita a metodologia utilizada neste estudo. A etapa seguinte apresenta a análise dos resultados. Por fim, na quinta parte, estão mencionadas as considerações finais, as limitações do estudo e as sugestões para novas pesquisas.

\section{REVISÃO DA LITERATURA}

A revisão da literatura inclui o levantamento de informações sobre os custos empresariais (conceitos gerais e classificações), a cadeia (processos da logística de suprimentos, de produção e de distribuição) e os custos logísticos (custos das etapas da cadeia logística) e modelos de mensuração de custos logísticos.

\subsection{Custos Empresariais}

Custos estão presentes em todos os tipos de empresas, sejam elas fabris ou industriais (Custos dos Produtos Vendidos), mercantis (Custo das Mercadorias Vendidas) ou de serviços (Custo dos Serviços Prestados) (BRUNI; FAMÁ, 2008). Os custos são os gastos relativos a todo trabalho realizado para produzir determinado bem ou serviço, desde que se atribua um valor monetário para esse trabalho (MARTINS, 2009; LEONE, 2000). Custo é o consumo associado ao sistema de elaboração do produto fabricado ou serviço prestado pela organização (BRUNI; FAMÁ, 2008). A alocação, interpretação e distribuição desses custos variam de empresa para empresa, tendendo a ficar mais complexas e detalhadas conforme a administração procura obter informações mais exatas para o auxílio à tomada de decisão (PEREIRA; AMARAL, 1998).

Há uma infinidade de classificações de custos, criadas para o atendimento de diferentes necessidades gerenciais, à medida que o administrador planeja ou toma decisão. Vários são os tipos de custos; tantos quantos forem tais necessidades. Dentre tantos tipos de custos, o auxílio à administração nos processos de planejamento e de tomada de decisões é realizado com o uso da classificação de variabilidade dos custos (fixos, variáveis e semivariáveis), o que implica análise da relação entre o valor total de um custo e o volume de atividade em determinado espaço de tempo (LEONE, 2000).

Os custos fixos são aqueles que independem das mutações da produção, pois os valores serão os mesmos para qualquer volume de produção, apesar de poderem variar com o decorrer do tempo. Os custos variáveis estão diretamente vinculados às alterações do volume de produção, pois seus valores aumentam à medida que se amplia a quantidade produzida. Os custos semivariáveis variam conforme o nível de produção, tendo uma parcela estabelecida mesmo que nada seja produzido, isto é, dispõem de uma parcela fixa e de uma parcela variável (MARTINS, 2009; BRUNI; FAMÁ, 2008; NEVES; VICECONTI, 2000; LEONE, 2000).

Além disso, os custos podem ser classificados em diretos ou indiretos, conforme sua aplicabilidade, ou seja, conforme sua relação com a unidade de produto ou de serviço. Custos diretos relacionam-se aos recursos diretamente empregados na produção, como materiais e mão de obra, 
sendo sua apropriação direta aos bens ou serviços produzidos. Custos indiretos demandam algum critério de rateio para serem apropriados, representando, em termos monetários, a capacidade instalada da empresa (MARTINS, 2009; BRUNI; FAMÁ, 2008; NEVES; VICECONTI, 2000; LEONE, 2000).

Martins (2009) e Neves e Viceconti (2000) consideram, ainda, a classificação de custos primários e custos de transformação, sendo os primários representados pela soma da matéria -prima com a mão de obra direta e os de transformação podem ser compreendidos como a soma de todos os outros custos de produção (que não incluam matéria-prima nem mão de obra) aplicados sem nenhuma modificação. O conjunto de indicações dos autores resulta no surgimento de parâmetros, definíveis em termos matemáticos, o que possibilita identificar relações utilizáveis em modelos de planejamento, de controle e de tomada de decisões (LEONE, 2000).

\subsection{A cadeia logística e os custos logísticos}

Logística relaciona-se a processo de planejamento, de execução e de controle do fluxo de bens e informações, desde o ponto de origem até o ponto de consumo, com a finalidade de atender às exigências dos clientes (Council of Logistics Management apud BALLOU, 2001). De maneira mais geral, pode-se identificar logística por meio da junção de quatro atividades básicas (aquisição, movimentação, armazenagem e entrega de produtos), pois seu objetivo é disponibilizar o produto certo, na quantidade certa, no local certo, no momento certo, nas condições adequadas para o consumo e a preço justo (FERRAES NETO; KUEHNE, 2002; CHRISTOPHER, 2007).

A cadeia logística envolve a integração das atividades de suprimentos, de produção e de distribuição. As atividades de suprimentos referem-se à busca e ao gerenciamento de recursos materiais a serem disponibilizados à produção; ao passo que as atividades de distribuição referem-se à oferta e ao gerenciamento dos bens produzidos aos consumidores (BALLOU, 2001). O alcance da logística perpassa toda a organização, visto que envolve desde o gerenciamento da matéria-prima até a entrega do produto final (CHRISTOPHER, 2007). Para Ballou (2001), a logística empresarial representa o elo físico entre a organização, os fornecedores e os clientes. Da empresa a montante, a logística trata de transportar, estocar, processar pedidos, armazenar, manusear materiais e manter informações com fornecedores, entre outras atribuições. Da empresa a jusante, as atividades são similares, contudo, o relacionamento é direcionado aos clientes, sejam eles consumidores intermediários ou finais. A abordagem logística deve analisar o modo de a administração aperfeiçoar os recursos de suprimento, os estoques e a distribuição dos produtos e serviços que a empresa disponibiliza ao mercado (POZO, 2004).

Uma vez que o gerenciamento logístico é um conceito orientado para o fluxo, com o objetivo de integrar recursos ao longo de toda a cadeia, é desejável que se defina um meio de avaliar este fluxo, por meio do uso de medidas matemáticas a fim de estabelecer a contribuição real do sistema logístico para a geração dos ganhos/perdas da empresa. Dentre essas medidas, pode-se apontar, por exemplo, equações de alocação de custos logísticos. Provavelmente, a falta de informações sobre custos logísticos seja um dos principais motivos para a dificuldade que muitas empresas enfrentam para adotar uma abordagem integrada. O problema da contabilização dos custos logísticos é complexo, porém, ainda assim, deve ser solucionado para que se possa desenvolver todo o potencial de gerenciamento logístico. Essencialmente, os problemas de apuração dos custos do gerenciamento logístico podem ser resumidos em: desconhecimento dos reais custos, levantamento de custos com alto grau de agregação, e uso de sistemas de rateio com base em custos gerais. A falta de visibilidade dos custos no fluxo logístico não fornece, aos gestores, uma informação confiável (CHRISTOPHER, 2002). 
Os custos associados à cadeia logística agrupam-se conforme sua adequação às etapas de suprimentos, de produção e de distribuição. Os custos de suprimentos incluem a aquisição de insumos (matéria-prima transformada no processo de produção), o transporte desses insumos e sua respectiva estocagem. Desse modo, as atividades de suprimentos incluem - de acordo com a realidade de cada organização - fabricantes, fornecedores, transportadores e depósitos, abrangendo-os em um fluxo de informações, produtos e capital. Em outras palavras, as atividades de suprimentos incluem suprir as necessidades de recursos materiais, planejar a demanda quantitativa, dispor das quantidades projetadas no momento certo, verificar o recebimento e providenciar armazenamento (DIAS, 1996; CHOPRA; MEINDL, 2003; BORBA; GIBBON, 2009). As origens dos suprimentos são bastante variadas, podendo ser classificadas quanto ao tipo de terminal, nos casos em que os suprimentos são transferidos a partir de outros meios de transporte (portos marítimos, aeroportos, pátios ferroviários, terminais intermodais); quanto ao tipo de fornecedor (indústria ou produtor, atacadista ou jazida da própria empresa) e quanto à geografia (estados, capitais, regiões do interior, municípios, países). Os destinos dos suprimentos são, em geral, as unidades de produção da empresa e os depósitos (ALVARENGA; NOVAES, 2000).

Para o custo da cadeia de suprimentos, compreendem-se o custo dos insumos (ou matérias-primas), o custo de aquisição e o custo de armazenagem desses insumos (DIAS, 1996; GOLDRATT, 2002; CHRISTOPHER, 2007). O custo dos insumos representa o desembolso feito pela empresa para a compra dos materiais necessários para alimentar seu processo produtivo. Seu resultado é diretamente proporcional à quantidade adquirida deste insumo, ou seja, é produto do custo unitário do insumo pelas quantidades compradas. O custo de aquisição, por sua vez, representa todo o gasto necessário para a realização do pedido de determinada quantidade de insumo, sendo composto por despesas com pessoal, despesas de materiais de consumo e outras despesas, tais como energia elétrica, telefone etc. (BORBA; GIBBON, 2009). O custo de armazenagem dos insumos refere-se aos gastos responsáveis por manter o produto estocado em depósitos da empresa e abrange os custos de armazenamento físico, de seguro, de transporte e manuseio dos insumos e de perdas (obsolescência), além de outros custos relacionados à estocagem. Seu valor final pode ser calculado a partir dos valores de quantidade pedida, dos custos dos insumos e dos custos de se manter insumos em estoque (BORBA; GIBBON, 2009). Deve-se salientar que há a possibilidade de que os estoques dos insumos fiquem sob a responsabilidade dos fornecedores, o que determina que tais custos não devam ser apropriados.

Os custos de produção consistem nos gastos incorridos no processo produtivo em determinado período de tempo, sendo, via de regra, incorporados aos estoques de produtos em processo (MARTINS, 2009). São, normalmente, compostos por três elementos distintos e frequentes: (a) material direto somado à (b) mão de obra direta e aos (c) custos indiretos de produção (NEVES; VICECONTI, 2000; MARTINS, 2009). Tais custos são mensurados monetariamente, registrados e, posteriormente, alocados, considerando-se critérios que visam possibilitar distintos tipos de decisões (PEREIRA FILHO; AMARAL, 1998). A composição do custo de produção é comum e generalizada a todas as atividades (LEONE, 2000).

Nesse sentido, material direto é todo material que pode ser identificado como unidade do produto que está sendo produzido. Geralmente, são constituídos pelas matérias-primas, pelas embalagens, pelos componentes adquiridos prontos e por outros materiais usados no processo de fabricação. Os materiais diretos incorporam-se ao custo dos materiais e incluem todos os gastos incorridos para sua colocação em condições de uso, caso seja adquirido para revenda (ou venda) ou para uso próprio (LEONE, 2000; MARTINS, 2009; BRUNI; FAMÁ, 2008). Por sua vez, a mão de obra direta refere-se aos esforços do pessoal que trabalha diretamente sobre o produto 
em fabricação ou sobre o serviço a ser prestado, uma vez que seja possível medir o tempo gasto e a identificação de quem realizou o trabalho, excluindo a necessidade de apropriação indireta ou de rateio (MARTINS, 2009). A mão de obra direta corresponde aos salários devidos aos operários e aos encargos sociais referentes ao conjunto de salários. Contudo, deve-se atentar aos elevados níveis de encargos sociais, visto que estes devem ser incorporados no custo do horário da mão de obra de forma variável (BRUNI; FAMÁ, 2008). Por fim, os custos indiretos de produção são os gastos relacionados com a função de produção, que não podem ser diretamente identificados com as unidades que estão sendo elaboradas, uma vez que não ocorrem homogeneamente em um determinado período. Os elementos dos custos indiretos de produção têm a finalidade de possibilitar que os materiais diretos, por meio da mão de obra direta, sejam transformados em novos produtos (MARTINS, 2009; BRUNI; FAMÁ, 2008).

Os custos de distribuição traduzem os gastos de todas as atividades relacionadas à transferência física do produto aos clientes. A organização da distribuição deve ser determinada com o objetivo de se obter uma adequada movimentação dos produtos acabados, dentro do menor custo operacional possível e obedecendo às diretrizes de um plano de ação previamente estabelecido pela administração (DIAS, 1996; CASTIGLIONI, 2009). Na distribuição física, os pontos de origem da mercadoria são constituídos pelas fábricas e pelos estoques, que podem ser próprios ou terceirizados. Os pontos de destino, por sua vez, podem ser classificados segundo os mesmos critérios dos pontos de origens dos suprimentos, definidos por Alvarenga e Novaes (2000), visto que as características dos processos de suprimentos e de distribuição são inversas. Assim, saída da distribuição é o carregamento, a troca de propriedade do produto e a transferência ou expedição propriamente dita (DIAS, 1996). Para o custo de distribuição, incluem-se o custo de armazenagem e o custo de transporte dos produtos prontos, além do custo dos produtos prontos vendidos (GOLDRATT, 2002; CASTIGLIONI, 2009).

O custo de armazenagem dos produtos prontos deve ser interpretado de maneira semelhante ao custo de armazenagem dos insumos: abrangendo os custos de armazenamento físico, de seguro, de perdas (obsolescência) e de manuseio dos produtos prontos, além de outros custos relacionados à manutenção de produtos em estoque. Devem ser considerados, no cálculo do custo de armazenagem, a multiplicação do custo unitário dos produtos vendidos, as quantias vendidas e os custos de estocagem de produtos prontos (BORBA; GIBBON, 2009). Por fim, o custo de transporte consiste nos gastos necessários para atender ao pedido de clientes e envolvem os desembolsos referentes à manutenção do modal utilizado pela empresa, ao pessoal e ao material de consumo. A empresa pode optar pelo serviço de entrega próprio e/ou, ainda, por um serviço de entrega terceirizado, cujo custo será representado pelo valor determinado pela empresa contratada, que fica responsável pela carga, por perdas e pelo cumprimento dos prazos, para realizar as entregas dos produtos prontos.

\subsection{Modelos de mensuração de custos logísticos}

Neste estudo, realizou-se, primeiramente, a descrição de custos, a cadeia e os custos logísticos, para, então, apresentar os modelos de mensuração de custos logísticos. Um modelo significa uma representação simplificada da realidade, estruturada de tal maneira que possibilite compreender o funcionamento total ou parcial dessa realidade, isto é, refere-se a um conjunto determinado de hipóteses sobre o comportamento de um fenômeno, baseadas em uma teoria já existente ou em novas suposições teóricas (MATOS, 1997). É possível construir um modelo muito menos complexo do que a realidade e, ainda assim, empregá-lo para prever e esclarecer 
fenômenos com determinado grau de precisão (SILVA et al., 1996; MATSUMOTO et al., 2006). Os modelos são usados, pelas organizações, como ferramentas para descrever e auxiliar operações de controle. Um bom modelo é aquele que tem desempenho suficientemente próximo do desempenho da realidade e é experimentável (SILVA et al., 1996). O uso de um bom modelo permite projetar experiências com maior eficiência e possibilita determinar como as alterações no sistema afetam seu desempenho (MATSUMOTO et al, 2006).

Várias associações e modelos podem ser elaborados com o objetivo de facilitar a percepção do comportamento dos custos, em diversos níveis e categorias. No entanto, existem modelos com maior ou menor aderência à mensuração de custos logísticos, uma vez que as componentes dos diferentes modelos variam em nomenclatura e abrangência. Vale salientar que, mesmo os autores que consideram seus modelos como modelos de mensuração de custos logísticos, não contemplam toda a cadeia logística, aludindo somente a segmentos do sistema logístico. Assim, este trabalho apresenta alguns dos modelos de apuração de custos, de forma que se perceba, em uma linha cronológica evolutiva, a ampliação de variáveis e de cobertura da cadeia logística, a partir de Uelze (1974), de Dias (1996), de Goldratt (2002), de Christopher (2007), de Castiglioni (2009) e de Borba e Gibbon (2009).

Figura 01 - Abrangência dos modelos de apuração de custos logísticos na cadeia logística

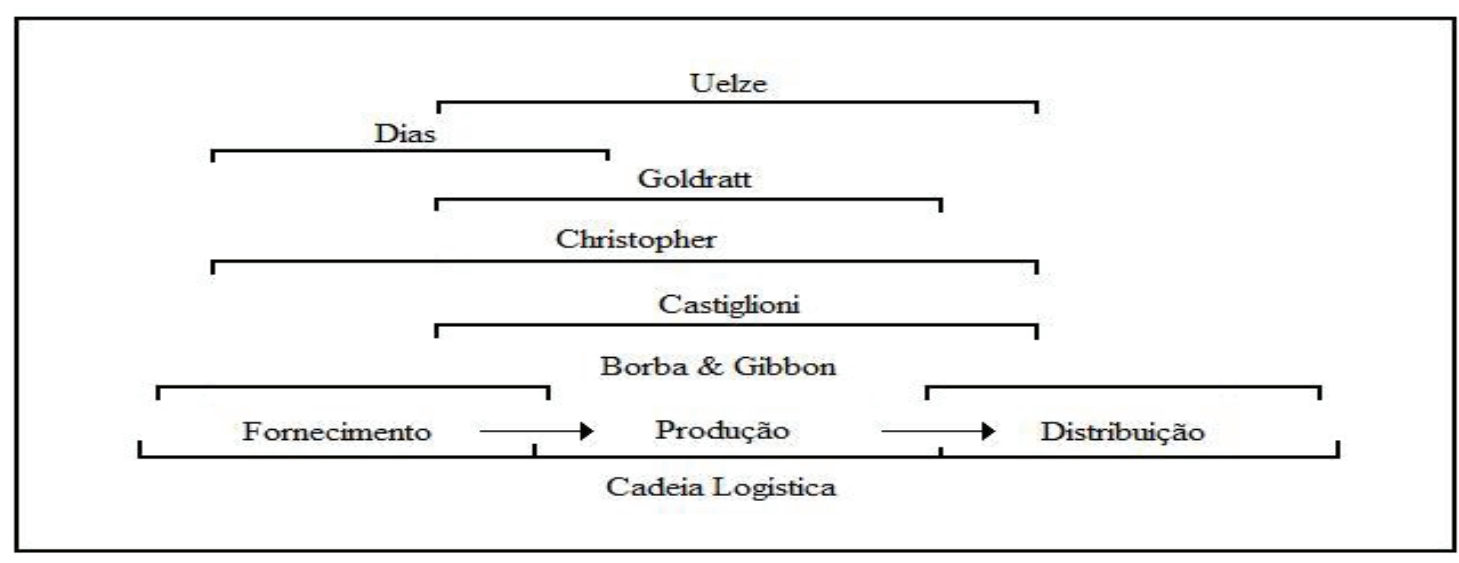

O modelo de Uelze (1974) indica que custo logístico (L) resulta do somatório de custo de transporte $(T)$ com o custo de armazenagem (A) mais o custo de manuseio do item (M) mais o custo de obsolescência $(O)$, considerando o período em que o produto estiver armazenado e em transporte, mais o custo do dinheiro (D), levando em consideração o tempo estocado e o tempo utilizado para o transporte do produto. $\mathrm{O}$ autor aponta que o papel do gestor da função logística é proporcionar meios (tempo e lugar) para que a organização atinja seus objetivos na agregação das utilidades. O estudioso identifica, ainda, algumas características que influenciam decisões logísticas, quanto à formação de custos, atendendo às várias características e aos diversos níveis de serviços viáveis em cada uma das áreas de atuação do agente logístico: características do risco (fragilidade, perecibilidade, periculosidade, alto valor ou possibilidade de ser roubada), uso de embalagens protetoras; volume de estoques a armazenar (definição de estoques de segurança), e nível de serviço logístico a ser oferecido.

O modelo de Dias (1996) mostra que o custo total (CT) é resultado da soma do custo total de armazenagem (CTA) com o custo total de pedido (CTP). Para o referido autor, a teoria de dimensionamento e controle de suprimentos objetiva em minimizar o custo total. O pesquisador afirma, ainda, que o custo de falta de estoque pode ocorrer quando um pedido atrasa ou não 
pode ser entregue pelo fornecedor, indicando formas de determinar o custo da ruptura (mas que não está contemplado no seu modelo de custo total). $O$ autor alega, também, que o custo total pode ser acrescido dos custos da distribuição, ou seja, dos gastos referentes aos esforços empregados para levar o produto aos consumidores, incluindo a manutenção de equipe de vendedores, as diversas rotas de entregas, no tempo e lugar certos, os riscos de concessão de crédito, as necessidades de capital circulante e a supervisão de vendas.

O modelo de Goldratt (2002) define que o custo de produção (P) é definido pela soma dos custos de estoque (E), que contemplam os custos de aquisição das matérias-primas e os custos de operação (O). Para o autor, os problemas estão centrados na logística de produção e, por consequência, em custos de produção. As ações para redução do custo total de produção dirigem-se à otimização do desempenho, o que determina a possibilidade de aplicação destas em outras áreas da empresa, como a logística de distribuição e o gerenciamento de projetos. Este modelo, entretanto, não foca toda a extensão da cadeia logística, uma vez que trabalha com a maximização do resultado das operações específicas de transformação, segundo as restrições de capacidades, sem contemplar todos os impactos dos custos de suprimentos e distribuição.

O modelo de Christopher (2007) indica que o custo total de uma rede de distribuição (CT) é composto pelo custo de transporte de materiais (CTr), custo de estocagem (CE), custo de depósitos (CD), custo de entregas locais (CEL) e custo de processamento de pedidos (CPP). O autor considera que um aspecto das decisões logísticas, que contribui para a complexidade de se gerar informação apropriada sobre custos, é que as decisões de custos geralmente são tomadas considerando-se o sistema logístico existente. Nesse contexto, a finalidade da análise de custo total é identificar a alteração ocasionada nos custos por essas decisões. O custo deve ser visto, deste modo, em termos incrementais - a mudança nos custos totais causada pela modificação no sistema.

O modelo de Castiglioni (2009) afirma que os custos logísticos (CL) são formados, basicamente, por quatro elementos distintos: o custo com processamento de pedidos (CPP), que se refere ao ressuprimento (compras) e à venda (pedido dos clientes); o custo com armazenagem (CA), referente ao acondicionamento dos bens e à movimentação destes; o custo com estocagem (CE), que é a soma dos custos de oportunidade de capital parado com custos com impostos e seguros, mais o risco de manter estoques e o custo com faltas; e o custo com transportes (CTr), que implica a movimentação do produto até o cliente. $O$ autor considera o custo de transporte como o mais importante na composição dos custos logísticos e divide-os em diretos ou indiretos, com subdivisão dos custos diretos em fixos e variáveis.

Por fim, o modelo de Borba e Gibbon (2009) mostra que custo logístico (CLog) é o somatório dos custos de suprimento (CS), que compreendem os gastos com aquisição e armazenagem de insumos; dos custos de distribuição (CD), que abarcam os gastos com a comercialização e armazenagem de produtos prontos e de outros custos $(\mathrm{OC})$ - termo aleatório que absorve os custos que necessariamente não estão contemplados aos custos de suprimento nem aos de distribuição, mas que são identificados como relevantes para a logística das organizações. Os autores não incluem os custos de produção, pois indicam que tais custos já são avaliados por outras formas de mensuração.

\section{METODOLOGIA}

Este trabalho configura-se como estudo exploratório, segundo a proposição de Cooper e Schindler (2003), uma vez que a investigação pretendida é nova ou ainda vaga, de forma que se necessita explorar o problema, porque variáveis importantes podem não ser conhecidas ou não estarem consolidadas. $\mathrm{O}$ estudo exploratório caracteriza-se como uma estratégia de pesquisa 
que visa examinar um fenômeno presente dentro de seu contexto, que pode trabalhar tanto com evidências qualitativas quanto quantitativas e que estimula novas descobertas (ROESCH, 1999). Este estudo exploratório foi executado por meio das seguintes etapas:

(1a) Definição do modelo de apuração de custos: Análise de estudos da literatura para indicar modelos de apuração de custos logísticos, considerando a cadeia logística em partes ou no todo. Por ser um estudo exploratório e centrado em Custos de Suprimentos, foi selecionado o modelo de Borba e Gibbon (2009), por este indicar os elementos necessários à captação de dados.

Figura 02 - Custos de Suprimento

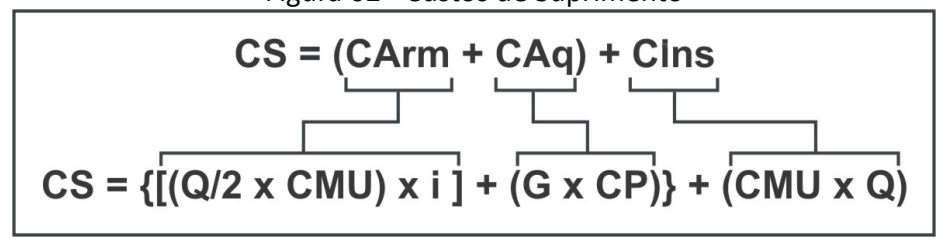

Fonte: Borba e Gibbon (2009).

(2a) Acesso aos dados de uma organização: Estabelecimento de contatos com empresas que apresentavam processos logísticos de suprimentos e que disponibilizassem dados quantitativos adequados ao modelo selecionado. $O$ processo de negociação, orientação e preparação dos dados ocorreu em 2010.

(3a) Formação da base de dados para atender às variáveis demandadas pelo modelo: As informações sobre custos de suprimentos, de um período de 12 meses, incluíram a descrição dos itens movimentados, a indicação da nomenclatura usada, os volumes quantitativos, o padrão de embalagens e a posição na Curva ABC.

(4a) Organização dos dados: As informações foram transferidas para tabelas eletrônicas (MS Excel ${ }^{\circledR}$ ). Cada tabela contemplou os dados fornecidos: giro por ano, quantidade adquirida por compra, padrão de embalagens, custo do item ou da mercadoria (CMerc), custo de reposição (CRep), nível de estoque médio, recursos financeiros (de capital) empregados, custo de posse ou armazenagem (CPos), formação do custo total mínimo (CTmín), composição do custo total anual (CTA) e Classificação $A B C$. O uso da classificação $A B C$ levou a tratamento e análise adicional dos dados coletados, por não estar incluso no modelo de apuração de custos, mas por ser uma prática corrente na organização, definindo ações gerenciais de controle e suporte para a definição de políticas de prioridades (DIAS, 1996; ARNOLD, 1999; VIANA, 2002; MARTINS; ALT, 2004).

(5a) Tratamento dos dados: Primeiramente, os itens foram organizados em uma planilha única, por ordem alfabética, contemplando as variáveis - giros, quantidades, custo unitário do item, custo por pedido (por rateio), custo de armazenagem (por rateio), custo total mínimo (custo de pedir mais custo de armazenar), custo total anual (custo do item mais custo total mínimo) e categorias da classificação $A B C$. Partindo da classificação $A B C$, organizaram-se os itens para verificar suas representatividades, em percentuais, quanto ao impacto nos custos. Em seguida, atualizaram-se os giros por ano de cada item, as quantidades adquiridas a cada compra e os custos.

(6a) Análise dos dados: Rearranjaram-se os itens em uma nova classificação $A B C$, considerando suas representatividades no custo total anual e no volume de itens. Além disso, avaliaram-se os dados relativos aos custos de suprimentos da cadeia logística, apontado medidas gerenciais para revisão dos processos de tomada de decisão. 


\section{RESULTADOS}

Os dados da organização, localizada na Região Sul do Rio Grande do Sul, foram organizados no formato sugerido por Borba e Gibbon (Modelo de Custos Logísticos, CONVIBRA, 2009). Embora o modelo inclua custos de suprimentos, custos de distribuição e outros custos, para este trabalho aplica-se apenas a mensuração dos custos logísticos de suprimentos, que incluem Custo da Mercadoria (CMerc: somatório de todos os itens que circularam pelo estoque e respectivos custos médios de contabilização), Custo de Reposição (CRep: custo derivado das estruturas do setor de compras em relação ao número de compras efetivado no ano), Custo de Posse (CPos: custo derivado das estruturas utilizadas para armazenamento) e Custo Total Anual (CTA: somatório dos custos de mercadoria, de aquisição e de armazenagem). Nesse sentido, foram utilizados 73 itens (correspondentes a $12 \%$ do total de insumos da empresa), que, conforme a classificação da Curva $A B C$, eram 15 da Classe A, 21 da Classe B e 37 da Classe C.

Os dados da Tabela 01 mostram que o Custo da Mercadoria (CMerc) totalizou R\$ 2.294.953,46, que, uma vez categorizado em percentuais ajustados, distribuiu-se em $71 \%$ (Classe A), 22\% (Classe B) e 7\% (Classe C). O Custo de Reposição (CRep) atingiu R\$ 151.440,00, categorizado em percentuais ajustados, indicou 39\% (Classe A), 25\% (Classe B) e 36\% (Classe C). O Custo de Posse (CPos) chegou a $\mathrm{R} \$ 23.333,18$, enquadrados em percentuais ajustados e distribuiu-se em 54\% (Classe A), 35\% (Classe B) e 12\% (Classe C). Por fim, o Custo Total Anual (CTA), que resulta da soma dos custos anteriores, indicou, em percentuais ajustados, $69 \%$ para a Classe $A, 22 \%$ para a Classe B e $9 \%$ para a Classe $C$.

Tabela 01 - Classes e custos dos itens

\begin{tabular}{|c|c|c|c|c|c|c|c|c|}
\hline \multirow{3}{*}{ Classes } & \multicolumn{8}{|c|}{ Custos Logísticos de Suprimentos } \\
\hline & \multicolumn{2}{|l|}{ CMerc } & \multicolumn{2}{|l|}{ CRep } & \multicolumn{2}{|l|}{ CPos } & \multicolumn{2}{|l|}{ CTA } \\
\hline & em RS & $\mathrm{em} \%$ & em RS & em $\%$ & em RS & em $\%$ & em RS & em $\%$ \\
\hline Classe A & $1.623 .946,47$ & 70,76 & $59.280,00$ & 39,14 & $12.558,99$ & 53,82 & $1.695 .785,46$ & 68,66 \\
\hline Classe B & $504.699,33$ & 21,99 & $38.280,00$ & 25,28 & $8.045,17$ & 34,48 & $551.024,50$ & 22,31 \\
\hline Classe C & $166.307,66$ & 7,25 & $53.880,00$ & 35,58 & $2.729,02$ & 11,70 & $222.916,68$ & 9,03 \\
\hline TOTAL & $2.294 .953,46$ & 100,00 & $151.440,00$ & 100,00 & $23.333,18$ & 100,00 & $2.469 .726,64$ & 100,00 \\
\hline
\end{tabular}

Fonte: Elaboração Própria.

Os Custos de Reposição e de Posse mostram-se como custos variáveis, visto que seus valores alteram-se conforme as quantidades adquiridas e armazenadas, respectivamente. À medida que o número de compras por período se reduz, a quantidade de insumos por compra aumenta, isto é, o valor do custo de reposição diminui ao passo que o valor do custo de posse aumenta. $O$ custo da mercadoria, por sua vez, foi considerado um custo fixo, quer pela prática de contabilização por custo médio unitário quer pela atual estabilidade econômica dos preços de aquisição.

Identificaram-se, também, outras situações que exigem atenção por parte dos gestores, visto que os giros (número de compras realizadas por ano) usados não estavam tecnicamente adequados à lógica de minimização e de equilíbrio entre os custos de pedir e de armazenar, que é a razão precípua de economicidade do modelo usado. Alguns dos 73 itens indicavam aquisições entre três e quatro vezes ao mês, mostrando-se desnecessárias e antieconômicas.

A Tabela 02 apresenta os seis elementos que evidenciaram as discrepâncias entre a situação vigente na empresa e a situação proposta (ajustada para obter equilíbrio entre custo de comprar e custo de armazenar), bem como a diferença quanto ao giro (G) de compras por ano e quantidade (Qtide) adquirida em cada compra. 
Tabela 02 - Situação atual, proposta e diferenças de giros e quantidades

\begin{tabular}{c|c|c|c|c|c|c}
\hline \multirow{2}{*}{ Itens } & \multicolumn{2}{|c|}{ Atual } & \multicolumn{2}{c|}{ Proposta } & \multicolumn{2}{c}{ Diferenças } \\
\cline { 2 - 7 } & G & Qtide & G & Qtide & G & Qtide \\
\hline no 64 & 48 & 8 & 4 & 92 & 44 & 84 \\
\hline no 14 & 48 & 3 & 5 & 32 & 43 & 29 \\
\hline no 08 & 40 & 9 & 7 & 54 & 33 & 45 \\
\hline n 10 & 40 & 10 & 7 & 57 & 33 & 47 \\
\hline no 12 & 36 & 4 & 4 & 40 & 32 & 36 \\
\hline no 38 & 39 & 10 & 7 & 57 & 32 & 47 \\
\hline
\end{tabular}

Fonte: Elaboração Própria.

A Tabela 03, abaixo, ilustra a situação atual e a alternativa proposta, indicando as diferenças de valores entre as duas condições, quanto aos custos de reposição, aos custos de posse e ao custo total mínimo. O Custo de Mercadoria não está incluso, pois, independentemente do número de giros, as quantidades serão adquiridas, para manter, no mínimo, os níveis de operação dos estoques. Observa-se que: (a) o custo de reposição poderia ser reduzido em até $72 \%$ em relação ao comumente usado, (b) o custo de posse poderia ser aumentado em até $76 \%$ em relação ao utilizado, e (c) o custo total mínimo, por sua vez, poderia ser reduzido em até $52 \%$, justificando a contribuição da proposta para que os administradores revejam seus processos de tomada de decisão.

Tabela 03 - Comparação entre a situação atual e a proposta, com as diferenças de custos

\begin{tabular}{c|c|c|c|c|c|c|c|c|}
\hline \multicolumn{3}{c|}{ Situação Atual (em R\$) } & \multicolumn{3}{c|}{ Proposta (em R\$) } & \multicolumn{3}{c|}{ Diferenças (em R\$) } \\
\hline Crep & Cpos & CTmín & Crep & Cpos & CTmín & Crep & \multicolumn{1}{c}{ Cpos } & CTmín \\
\hline $\mathbf{1 5 1 . 4 4 0 , 0 0}$ & $\mathbf{2 3 . 3 3 3 , 1 8}$ & $\mathbf{1 7 4 . 7 7 3 , 1 8}$ & $\mathbf{4 1 . 7 6 0 , 0 0}$ & $\mathbf{4 1 . 1 1 0 , 8 8}$ & $\mathbf{8 2 . 8 7 0 , 8 8}$ & $\mathbf{- 1 0 9 . 6 8 0 , 0 0}$ & $\mathbf{1 7 . 7 7 7 , 7 0}$ & $\mathbf{- 9 1 . 9 0 2 , 3 0}$ \\
\hline
\end{tabular}

Fonte: Elaboração Própria.

Constatou-se, ainda, falta de atualização na aplicação da classificação $A B C$, dado que alguns itens de baixo valor ainda estavam enquadrados nas classes A e B. Decidiu-se, então, reorganizar os itens em uma nova classificação $A B C$ (Tabela 04), a partir da alternativa proposta. A Tabela 04 mostra a reorganização dos itens e os novos custos obtidos a partir das classes $A B C$ propostas.

Tabela 04 - Reorganização dos itens e dos custos na nova Curva ABC

\begin{tabular}{c|c|c|c|c|c|c|c|c|c}
\hline \multirow{2}{*}{ Classes } & \multicolumn{4}{|c|}{ Qtide de Itens } & \multicolumn{4}{c}{ Custos da Proposta (em R\$) } \\
\cline { 2 - 10 } & Atual & $\%$ & Proposta & $\%$ & $\Delta \%$ & CMerc & CR & CP & CTA \\
\hline A & 15 & 20,55 & 6 & 8,22 & $-12,33$ & $1.511 .174,05$ & $13.920,00$ & $14.031,04$ & $1.539 .125,09$ \\
\hline B & 21 & 28,77 & 21 & 28,77 & 0,00 & $678.967,51$ & $17.640,00$ & $17.736,08$ & $714.343,59$ \\
\hline C & 37 & 50,68 & 46 & 63,01 & 12,33 & $104.811,89$ & $10.200,00$ & $9.343,77$ & $124.573,33$ \\
\hline TOTAL & $\mathbf{7 3}$ & $\mathbf{1 0 0 , 0 0}$ & $\mathbf{7 3}$ & $\mathbf{1 0 0 , 0 0}$ & $\mathbf{x x}$ & $\mathbf{2 . 2 9 4 . 9 5 3 , 4 5}$ & $\mathbf{4 1 . 7 6 0 , 0 0}$ & $\mathbf{4 1 . 1 1 0 , 8 9}$ & $\mathbf{2 . 3 7 7 . 8 2 4 , 3 4}$ \\
\hline
\end{tabular}

Fonte: Elaboração Própria.

A nova classificação agrupa, conforme os valores de custo total anual (CTA) dos suprimentos, tendo, como critério, três faixas de valores que correspondem, respectivamente, às Classes $C(R \$ 0,00$ até $R \$ 10.000,00), B$ (maior que $R \$ 10.000,00$ e até $R \$ 100.000,00)$ e $A$ (valores acima de $R \$ 100.000,00)$. O resultado desta Curva $A B C$ mostra que: (a) a Classe $A$, que inicialmente contava com 15 itens, passou a ter apenas seis, o que representa uma redução de $12,33 \%$ no percentual de itens da classe; (b) a Classe B não apresentou variação no número de itens, 
mantendo-se com 21; e (c) a Classe C passou de 37 para 46 itens, ampliando-se em 12,33\%, por deslocamento de itens que anteriormente enquadravam-se na Classe A. Isto implica menor número de itens sobre os quais devem ser aplicados esforços de controle, além de uma redução de $\mathrm{R} \$$ 91.902,30, gerada pelo tratamento das variáveis dos custos de aquisição e de armazenagem, ou seja, pela otimização dos giros e pelo equilíbrio dos custos.

\section{CONSIDERAÇÕES GERAIS}

Este estudo objetivou a avaliação de custos de suprimentos com a aplicação de um modelo de mensuração de custos logísticos em uma empresa de grande porte. O modelo aplicado mostrou, na condição original, que estavam sendo aplicados cerca 2,5 milhões de reais na gestão dos suprimentos, com custos de reposição e de armazenagem em desequilíbrio, indicando um esforço de obtenção superior ao de armazenagem. Readequando os dados da empresa, é possível obter redução nos custos de reposição e armazenamento de suprimentos na ordem de $52 \%$.

Ainda, levando em consideração que a amostra estudada representa cerca de $12 \%$ dos estoques no exercício, pode-se inferir que estes valores podem sofrer acréscimos significativos, dada a distorção de classificação na Curva ABC. Dessa forma, o modelo de aplicado respondeu à proposta exploratória do trabalho, tendo em vista que foi possível tratar dos custos de suprimentos, pois, além de indicar resultados econômico-financeiros, evidencia as exigências de mudanças técnicas e culturais na empresa. Nesse sentido, o modelo dispõe de elementos para intervir e auxiliar na forma de gestão e de tomada de decisão na área de logística de suprimentos, demonstrando as condições de operação quanto aos gastos de insumos, de aquisição e de armazenagem.

Nessa perspectiva, percebe-se que os custos logísticos representam um dos principais elementos para a definição das estratégias de suprimentos, desde que devidamente mensurados e gerenciados. Isso leva a recomendar, à organização, ações para limitar os problemas gerenciais de gestão de suprimentos, tais como: (a) rever as práticas de definição de giro de compras ao ano, a fim de reduzir os custos provenientes das estruturas de compras, sem descuidar dos impactos a serem transferidos à área de armazenagem; (b) capacitar recursos humanos para entender e buscar o equilíbrio entre custos de aquisição e custos de estocagem; (c) desenvolver, simultaneamente, práticas de contabilidade gerencial, a fim de manter controle permanente sobre o conjunto de variáveis dos custos de suprimentos; (d) definir a forma de atualização, a intervalos regulares, da classificação $A B C$, visando aplicar controles diferenciados aos estoques; (e) comparar, periodicamente, os custos de suprimentos com seu enquadramento nas classes da Curva $A B C$, para otimizar resultados; (f) complementar a análise da Curva $A B C$, aplicando informações de criticidade de uso de itens, conforme os agrupamentos em famílias e/ou setores da organização; (g) registrar permanentemente os dados coletados, visando análise de custos; e, (h) avaliar modelo de mensuração de custos logísticos que atenda às características e às necessidades da empresa.

Como uma das principais limitações do estudo, aponta-se a escassa cultura organizacional para aplicação da contabilidade de custos, no setor de logística, devido à falta de dados para mensurar os custos de toda a cadeia logística. Além disso, verificou-se que não há continuidade no levantamento de dados para que um modelo de custos logísticos possa ser aplicado sistematicamente. Por conta da amplitude de dados de custos logísticos, a empresa apenas cumpre as exigências contábeis, as quais aliam técnicas simplificadas (como é o caso da classificação $A B C$ ), o que gera inúmeros problemas gerenciais, tanto pela falta de atualização continuada de informações como pelas consequentes inadequações na gestão de seus suprimentos.

Por fim, percebem-se possibilidades para futuros trabalhos, que poderão testar as ou- 
tras variáveis do modelo de mensuração de custos logísticos, a partir de organizações que operem a cadeia logística de forma integrada, ou, até mesmo, segmentada em atividades de suprimentos ou de distribuição.

\section{REFERENCIAS}

ALVARENGA, A. C.; NOVAES, A. G. Logística Aplicada: suprimento e distribuição física. 3a ed. São Paulo: Blücher, 2000.

ARNOLD, J. R. T. Administração de Materiais. São Paulo: Atlas, 1999.

BALLOU, R. H. Gerenciamento da Cadeia de Suprimentos: planejamento, organização e logística empresarial. 4a ed. Porto Alegre: Bookman, 2001.

BORBA, J. V. S.; GIBBON, A. R. Modelo de Custos Logísticos (2009). In: VI CONVIBRA Congresso Virtual Brasileiro de Administração. Disponível em: http://www.convibra.com. br/2009/artigos/118_0.pdf. Acesso: 10 abr. 2011.

BRUNI, A. L.; FAMÁ, R. Gestão de Custos e Formação de Preços. 5a ed. São Paulo: Atlas, 2008.

CASTIGLIONI, J. A. M. Logística Operacional: um guia prático. 2a ed. São Paulo: Érica, 2009.

CHOPRA, S.; MEINDL, P. Gerenciamento da Cadeia de Suprimentos: estratégia, planejamento e operação. São Paulo: Pearson Prentice Hall, 2003.

CHRISTOPHER, M. Logística e Gerenciamento da Cadeia de Suprimentos: estratégias para a redução de custos e melhoria dos serviços. São Paulo: Pioneira Thomson Learning, 2002.

. Logística e Gerenciamento da Cadeia de Suprimentos: criando redes que agregam valor. 2a ed. São Paulo: Thomson Lerning, 2007.

COOPER, D. R.; SCHINDLER, P. Métodos de Pesquisa em Administração. 7ạ ed. Porto Alegre: Bookman/Artmed, 2003.
DIAS, M. A. P. Administração de Materiais: uma abordagem logística. 4a ed. São Paulo: Atlas, 1996.

FERRAES NETO, F.; KUEHNE JUNIOR, M. Gestão Empresarial. Curitiba: Associação Franciscana de Ensino Senhor Bom Jesus, 2002. v. 2. cap. 4.

GOLDRATT, E.; COX, J. A meta: um processo de melhoramento contínuo. 2a ed. São Paulo: Nobel, 2002.

LEONE, G. S. G. Custos: planejamento, implantação e controle. 3 a ed. São Paulo: Atlas, 2000.

LOPES, L. F. L.; MACHADO, F. P.; LOPES, F. G.; LIMA, M. P.; COSTAS, V. M. F.; SILVA, D. D. M. Uma análise do ambiente mercadológico de um a empresa do setor metal mecânico sob as perspectivas de Porter. Revista de Administração da UFSM, v. 6, n. 1, p.103-118, jan/mar, 2013.MARTINS, E. Contabilidade de custos. 9a ed. São Paulo: Atlas, 2009.

MARTINS, P. G.; ALT, P. R. C. Administração de Materiais e Recursos Patrimoniais. São Paulo: Saraiva, 2004.

MATOS, O. C. Econometria Básica: teoria e aplicações. 2a ed. São Paulo: Atlas, 1997.

MATSUMOTO, A. S.; PEREIRA, S. E.; NASCIMENTO, G. S. A utilização da Contabilometria e a agregação de valor à informação contábil (2006). In: 30 Congresso USP de Iniciação Científica em Contabilidade. São Paulo. Disponível em: www.congressousp. fipecafi.org/artigos32006/255.pdf. Acessado em mar/2010.

NEVES, S.; VICECONTI, P. E. V. Contabilidade de Custos: um enfoque direto e objetivo. 6a ed. São Paulo: Frase, 2000.

PEREIRA Fo, A. D.; AMARAL, H. F. A Contabilidade 
de Custos como instrumento de informação gerencial - um enfoque no sistema de custeio ABC (1998). In: Contabilidade Vista e Revista. Belo Horizonte. v. 9. n. 2.

PIANA, J.; ERDMANN, R. H. Fatores geradores de competitividade na manufatura: uma relação entre práticas e resultados. Revista de Administração da UFSM, v. 4, n.1, p. 73-90, jan./abr. 2011.

POZO, H. Administração de Recursos Materiais e Patrimoniais: uma abordagem logística. 3a ed. São Paulo: Atlas, 2004.

ROESCH, S. M. Projetos de estágio e de pesquisa em administração. 2a ed. São Paulo: Atlas, 1999.

SILVA, E. M.; SILVA, E. M.; GONÇALVES, V.; MUROLO, A. C. Pesquisa Operacional. 2a ed. São Paulo: Atlas, 1996.

UELZE, R. Logística Empresarial: uma introdução à administração dos transportes. São Paulo: Pioneira, 1974.

VIANA, J. J. Administração de Materiais: um enfoque prático. São Paulo: Atlas, 2002. 